\section{Benefit v. risk when using chloroquine in patients with severe COVID-19 disease}

To the Editor: Chloroquine (CQ) is widely advocated as treatment for coronavirus disease 2019 (COVID-19), including the president of the USA publicly supporting the use of hydroxychloroquine (HCQ) as a 'game-changer' on the social media platform Twitter. CQ and HCQ are structurally similar, with HCQ having an $N$-hydroxyl-ethyl side-chain in place of the $\mathrm{N}$-diethyl group. ${ }^{[1]}$ Currently only CQ is being marketed in South Africa. We encourage the development of curative directed therapy against severe acute respiratory syndrome coronavirus 2 (SARS-CoV-2) using appropriate designed trials and regulatory oversight, and caution against the indiscriminate use of CQ or HCQ. Careful patient selection is essential, including assessing prognosis, anticipated benefit and potential harms prior to initiating CQ/HCQ therapy.

The benefit of CQ/HCQ is not yet clearly defined. CQ/HCQ demonstrated in vitro antiviral activity against SARS-CoV-2..$^{[2,3]}$ Although the antiviral mechanisms of CQ/HCQ are still being investigated, a number of mechanisms have been proposed. CQ/ HCQ inhibits autophagy by impairing membrane fusion of SARS$\mathrm{CoV}-2$ with intracellular endosomes or lysosomes, perhaps by increasing the $\mathrm{pH}$ of these organelles; ${ }^{[4]}$ interferes with glycosylation intracellular receptors (angiotensin-converting enzyme 2); ${ }^{[5,6]}$ or inhibits T-cell-mediated proinflammatory cytokines implicated in acute respiratory distress syndrome. ${ }^{[7]}$ In vivo evidence is currently limited but increasing with multiple ongoing studies. A small French open-label non-randomised controlled trial of 26 patients diagnosed with COVID-19 receiving HCQ is widely cited.$^{[8]}$ Of the 26 patients, only 20 were included in the analysis; 6 were receiving concomitant azithromycin for bacterial infections. Sixteen control patients were included. Viral clearance on nasopharyngeal swabs was greater at day 6 in HCQ and greatest in the 6 HCQ plus azithromycintreated patients. Although promising, the study suffered from many limitations, as outlined by Dahly et al..$^{[9]}$

Others have argued that our understanding of the SARS-CoV-2 pathophysiology is incomplete and that the immune effects of CQ/ HCQ are unknown. ${ }^{[10]}$ CQ had a paradoxical effect, where the decrease in cytokines delayed the adaptive immune response with worsened disease in a primate study with Chikungunya virus. ${ }^{[1]}$ Although CQ and HCQ have been used safely for decades to treat outpatients with rheumatic diseases and malaria, critically ill patients with severe SARS-CoV-2 disease and immune dysregulation may be at higher risk of CQ/HCQ toxicity. The toxic dose of $\mathrm{CQ}$ and HCQ is close to the therapeutic range. ${ }^{[12,13]} \mathrm{CQ} / \mathrm{HCQ}$ cardiotoxicity includes dysrhythmias, depressed cardiac contractility and conduction associated with hypokalaemia due to potassium shifting. Renal and hepatic impairment may increase CQ/HCQ concentrations, as CQ and HCQ are renally eliminated and hepatically metabolised. ${ }^{[14]}$ Underlying cardiac disease or cardiac risk factors, concomitant QT-prolonging medicines and enzyme inhibitors may therefore increase the risk of $\mathrm{CQ} / \mathrm{HCQ}$ toxicity. Investigational therapy, treatment or prophylaxis, with unknown benefit v. harm, is best studied as part of a clinical trial with appropriate ethical and regulatory oversight. Lastly, CQ stock is limited worldwide, and CQ is an essential medicine to treat systemic lupus erythematosus (SLE). Stock prioritisation away from these patients may lead to acute disease flare-ups, which could add additional pressure to the health system and unnecessarily expose SLE patients to the hospital environment.

\section{Eric H Decloedt, Helmuth Reuter}

Division of Clinical Pharmacology, Department of Medicine, Faculty of Medicine and Health Sciences, Stellenbosch University and Tygerberg Hospital, Cape Town, South Africa ericdecloedt@sun.ac.za

\section{Brian Allwood}

Division of Pulmonology, Department of Medicine, Faculty of Medicine and Health Sciences, Stellenbosch University and Tygerberg Hospital, Cape Town, South Africa

\section{Arifa Parker}

Division of Infectious Diseases, Department of Medicine, Faculty of Medicine and Health Sciences, Stellenbosch University and Tygerberg Hospital, Cape Town, South Africa

\section{Coenraad F N Koegelenberg}

Division of Pulmonology, Department of Medicine, Faculty of Medicine and Health Sciences, Stellenbosch University and Tygerberg Hospital, Cape Town, South Africa

\section{Marc Blockman}

Division of Clinical Pharmacology, Department of Medicine, Faculty of Health Sciences, University of Cape Town and Groote Schuur Hospital, Cape Town, South Africa

\section{Jantjie Taljaard}

Division of Infectious Diseases, Department of Medicine, Faculty of Medicine and Health Sciences, Stellenbosch University and Tygerberg Hospital, Cape Town, South Africa

1. Sahraei Z, Shabani M, Shokouhi S, Saffaei A. Aminoquinolines against coronavirus disease 2019 (COVID-19): Chloroquine or hydroxychloroquine. Int J Antimicrob Agents 2020 (epub 17 March 2020). https://doi.org/10.1016/j.ijantimicag.2020.105945

2. Liu J, Cao R, Xu M, et al. Hydroxychloroquine, a less toxic derivative of chloroquine, is effective in inhibiting SARS-CoV-2 infection in vitro. Cell Discov 2020;6:16. https://doi.org/10.1038/s41421020-0156-0

3. Yao X, Ye F, Zhang M, et al. In vitro antiviral activity and projection of optimized dosing design of hydroxychloroquine for the treatment of severe acute respiratory syndrome coronavirus 2 (SARShydroxychloroquine for the treatment of severe acute respiratory syndrome coronavis

4. Mauthe M, Orhon I, Rocchi C, et al. Chloroquine inhibits autophagic flux by decreasing Mauthe M, Orhon I, Rocchi C, et al. Chloroquine inhibits autophagic flux by decreasing
autophagosome-lysosome fusion. Autophagy 2018;14(8):1435-1455. https://doi.org/10.1080/1554

5. Savarino A, di Trani L, Donatelli I, Cauda R, Cassone A. New insights into the antiviral effect of chloroquine. Lancet Infect Dis 2006;6(2):67-69. https://doi.org/10.1016/S1473-3099(06)70361-9 6. Zhang H, Penninger JM, Li Y, Zhong N, Slutsky AS. Angiotensin-converting enzyme 2 (ACE2) as a SARS-CoV-2 receptor: Molecular mechanisms and potential therapeutic target. Intensive Care Med 2020;46:586-590. https://doi.org/10.1007/s00134-020-05985-9

7. Landewé RB, Miltenburg AM, Verdonk MJ, et al. Chloroquine inhibits T cell proliferation by interfering with IL-2 production and responsiveness. Clin Exp Immunol 1995;102(1):144-151. https://doi.org/10.1111/j.1365-2249.1995.tb06648.x

8. Gautret P, Lagier I-C, Parola P, et al. Hydroxychloroquine and azithromycin as a treatment of COVID-19: Results of an open-label non-randomized clinical trial. Int J Antimicrob Agents 2020 (epub 20 March 2020). https://doi.org/10.1016/j.ijantimicag.2020.105949

9. Dably D, Gates S, Moris T Statistical review of hydroxychloroquine and azithomycin as a Dahly D, Gates S, Moris T. Statistical review of hydroxychloroquine and azthromycin as 2020. hips//doiorg $10.5281 / Z$ ENOD 3725560

10. Guastalegname M, Vallone A. Could chloroquine/hydroxychloroquine be harmful in coronavirus disease 2019 (COVID-19) treatment? Clin Infect Dis 2020 (epub 24 March 2020). https://doi org/10.1093/cid/ciaa321

11. Roques P, Thiberville S-D, Dupuis-Maguiraga L, et al. Paradoxical effect of chloroquine treatment in enhancing Chikungunya virus infection. Viruses 2018;10(5):268. https://doi.org/10.3390/v1005026 2. Good MI, Shader RI. Lethality and behavioral side effects of chloroquine. J Clin Psychopharmaco 1982;2(1):40-47. https://doi.org/10.1097/00004714-198202000-00005

3. Abdulaziz N, Shah AR, McCune WJ. Hydroxychloroquine: Balancing the need to maintain therapeutic levels with ocular safety: An update. Curr Opin Rheumatol 2018;30(3):249-255. https:// doi.org/10.1097/BOR.0000000000000500

14. Browning DJ. Pharmacology of chloroquine and hydroxychloroquine. In: Hydroxychloroquine and Chloroquine Retinopathy. New York: Springer, 2014:35-63. https://doi.org/10.1007/978-1-4939$0597-3-2$

S Afr Med J 2020;110(5):336. https://doi.org/10.7196/SAMJ.2020.v110i5.14761 